\title{
Power Quality Prediction Based on BP Neural Network
}

\author{
Yanzhen Li ${ }^{1, \text { a }}$, Yinglei Guo ${ }^{1, b}$, Pengfei Shen ${ }^{2, ~ c}$, Yifeng Deng ${ }^{2, ~ d}$, and Kaiqiang Cheng ${ }^{2, ~ e ~}$ \\ ${ }^{1}$ State Grid Qingdao Power Supply Company, Qingdao 266002, China. \\ ${ }^{2}$ School of electrical engineering, Shandong University, Jinan 250061, China. \\ a)4507877@qq.com, ${ }^{b)}$ gyinglei@163.com, ${ }^{c)}$ Corresponding \\ author:1477758543@qq.com, ${ }^{d)}$ 17853138876@163.com, ${ }^{e}$ 1061386058@qq.com
}

Keywords: Power Quality, BP Neural Network, Prediction

\begin{abstract}
With the large-scale use of nonlinear loads in power grid, power quality issues are becoming increasingly prominent. However, the widespread application of sensitive loads has made research on power quality prediction a hot topic. This paper proposes a power quality prediction method based on BP neural network. Firstly, the paper theoretically analyzes the BP neural network, then establishes a power quality prediction model; finally, the MATLAB program was designed and written to be applied to two different data samples to test and analyze the prediction results. The simulation results show that when there are more historical data to train BP neural network, this method has a good predictive effect on power quality indicators.
\end{abstract}

\section{Introduction}

With the development of industrialization, the non-linear, impact and other disturbance loads in the power system have increased dramatically, making power quality problems more and more prominent. The importance of power companies to power quality issues has also been continuously improved, and the construction of power quality online monitoring systems has been gradually improved. The monitoring platform collects a large amount of power quality monitoring data every day, and there are still many shortcomings in the application of these data ${ }^{[1]}$.

Power quality prediction is based on the analysis of the changing rules about the monitoring data, fully exploiting the potential relationship between historical data. By finding the law of data change, we can predict the power quality indicators in the future, so as to find potential power quality problems ahead of time. Operation and maintenance personnel can take timely measures, so as to minimize the various problems caused by power quality, and maintain the safe and stable operation of power grid ${ }^{[2]}$.

The reference [3] proposes a prediction method based on Monte Carlo sampling. The load current data could be gotten with the Monte Carlo model according to the probability distribution models. [4] uses the ARIMA time series algorithm to predict the active power, and establishes neural network model to predict conventional indicators according to the relationship between active power and power quality indicators. The reference [5] presents a hybrid model with the kmeans clustering and BP neural network to forecast the electricity load based on the existing electric power data.

Most of the predictions of power quality in the references have combined the knowledge of 
probability and statistics. Most of the prediction models are linear, and the prediction results are deviated from the actual values. In this paper, power quality prediction based on BP neural network algorithm is proposed, and artificial intelligence algorithm is adopted to improve the prediction accuracy. The BP neural network are introduces in section 1; the power quality prediction model is established in section 2; section 3 proves the accuracy of the algorithm through the examples; finally, the main conclusion are drawn in section 4 .

\section{BP neural network algorithm}

\subsection{Multilayer Feed-forward Neural Network Model}

The neural network model is made up of many interconnected neuron models. A basic neuron model consists of three parts: input, computation and output. A neuron model can receive multiple inputs. Each input quantity is multiplied by a corresponding weight and then summed, and the obtained sum is added with an offset quantity, then processed by a nonlinear function, and the value of the obtained function is the final output.

Multiple neuron models are arranged hierarchically and connected to each other according to certain principles, and multilayer neural networks can be constructed. A three-layer neural network model is shown in figure 1. From left to right are the input layer, the hidden layer, and the output layer. The neurons in each layer of the multilayer neural network are not connected to each other. Each layer of neurons is connected to the next layer of neurons, and the output of the previous layer is the input of the latter layer. The input is processed from the input layer through one or more hidden layers and finally output by the output layer. This one-way multi-layer neural network is a feedforward neural network model.

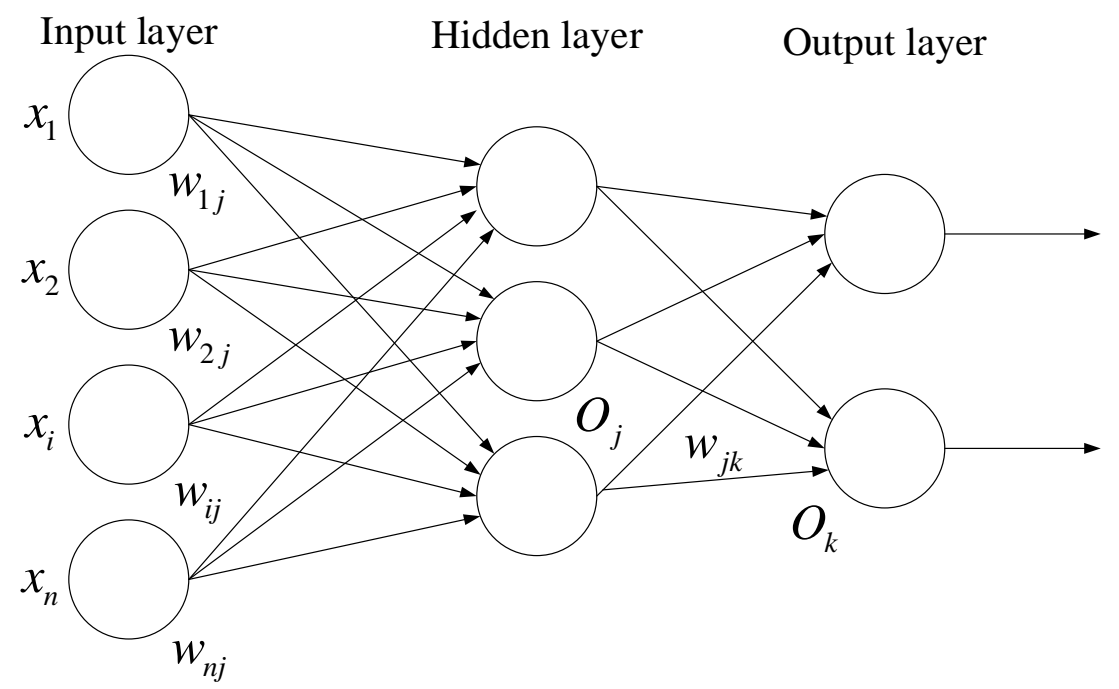

Figure 1. Three-layer neural network model.

The weight of each connection between the layers in the feedforward neural network model, and the base offset of each neuron, ie the threshold, are undetermined. Through the existing historical data, that is, the training samples, the weights and thresholds of the neural network are continuously updated according to a certain learning algorithm. Finally, a neural network model that performs well on both the training sample and the test sample is obtained, that is, the trained neural network model. 


\subsection{Back Propagation Algorithm}

Back propagation algorithm, referred to as BP algorithm, is a commonly used neural network training method. During data sample training, the input data is passed to the input layer of the neural network, and processed through the hidden layer and the output layer to obtain an output value. Then the actual output value is compared with the expected output value to get the error. This process is forward propagation.

The commonly used error measure is the average squared error, which is obtained by equation 1:

$$
E=\frac{1}{2 m} \sum_{\mu}\left(y_{\mu}-\hat{y}_{\mu}\right)^{2}
$$

In the formula, $\mathrm{m}$ is the number of training samples; $y_{\mu}$ is the expected output of a training sample; and $\hat{y}_{\mu}$ is the actual output of the training sample after processing through the current network.

The error function E can be expressed as a function of weights and thresholds, and the partial derivatives of the error functions to the weights and thresholds of each connection are obtained. The opposite number of partial derivative, that is, the negative gradient direction of function, is the fastest direction of error reduction. On the basis of each of the original weights and thresholds, plus the value of the corresponding negative partial derivative function, the updated weight and threshold are obtained.

Through the above method, the error function is reversely transmitted from the output layer to the input layer, and the weight and threshold of each layer are adjusted according to the negative gradient direction. This process is called a back propagation process. The purpose of the back propagation process is to reduce the function value of the error function, and constantly adjust the parameters of the neural network to optimize the parameters.

\subsection{BP Neural Network}

The multilayer feedforward neural network trained by BP algorithm is BP neural network. The training process of the BP neural network is to adjust the parameters through forward calculation and back propagation for each sample. Performing such training on all the data in the training sample will result in a trained neural network. The trained neural network needs to be verified on the validation samples and tested by the test samples to test its performance and generalization ability. 


\section{Power quality prediction model}

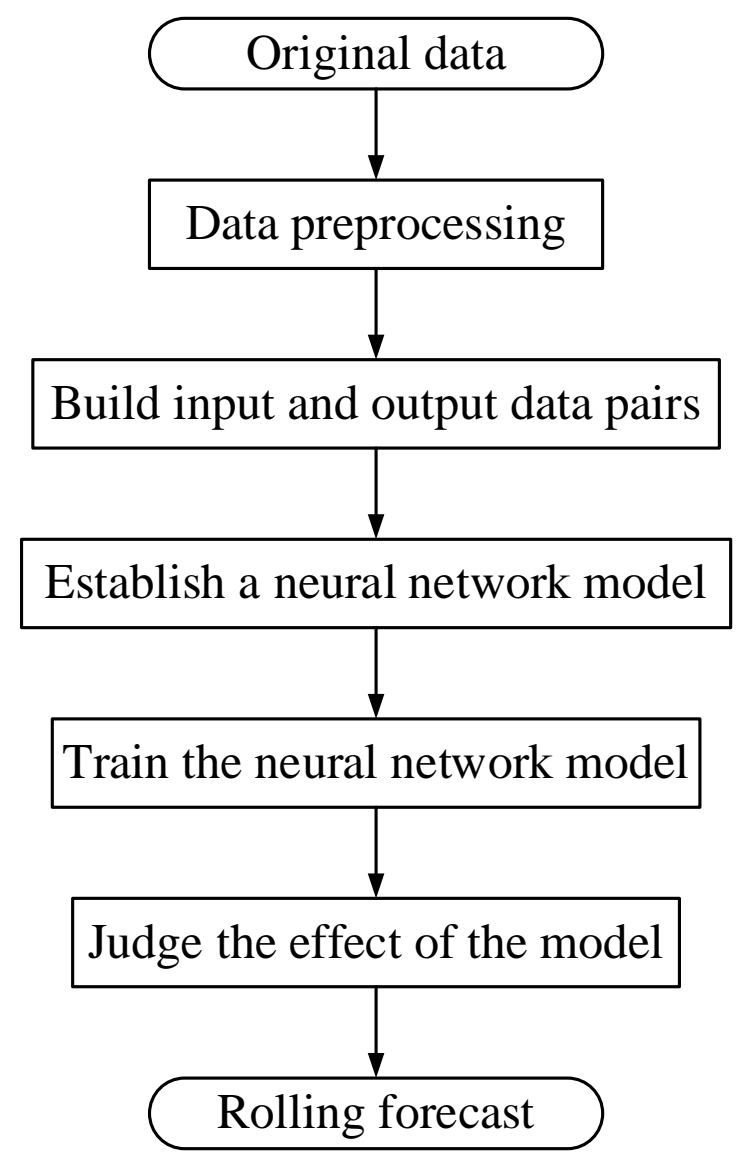

Figure 2. Neural network prediction flow chart.

The process of predicting power quality indicator data using BP neural network model is as follows.

Step 1: Data preprocessing. Read the original data and divide them into two parts: learning sequence and prediction sequence.

Step 2: Build input and output data pairs. The autoregressive order $\mathrm{n}$ is determined, that is, the values of the previous $n$ moments are used to predict the value of the next moment. And the input and output data pairs are constructed based on the learning sequence.

Step 3: Establish a neural network model. Determine the number of neural network layers and the number of hidden layer neurons, and use MATLAB software to write functions to create neural networks.

Step 4: Train the neural network model. The learning sequence is divided into three parts: training sample, verification sample and test sample. After setting the training parameters, call the function to train.

Step 5: Judge the effect of the model. The autocorrelation, partial correlation and error of the model are viewed through the neural network toolbox.

Step 6: Rolling forecast. The predictive value of future time is obtained by rolling prediction using trained neural network model.

The programming flow chart for prediction using the BP neural network model is shown in figure 2 . 


\section{Case study}

\subsection{The Introduction of Example Data}

In this paper, the power quality data is predicted based on the data of the 5th harmonic voltage and the voltage deviation of a monitoring point. The 5th harmonic voltage contains 29 data points in days, and the voltage deviation is 310 nodes in minutes. In order to test the effect of the model prediction, the historical data is divided into two parts: the training sample and the test sample. Training samples are used for model building and parameter estimation, and test samples are used to test the effects of model predictions.

The first example is the prediction of the 5th harmonic voltage ratio. The training sample is the data of the first 25 time points, and the predicted sample is the data of the last 4 time points. This data size is small. The second example is the prediction of the voltage deviation. The training sample is the first 300 time points, and the prediction sample is the data of the last 10 time points. This data is large in scale.

\subsection{Prediction Results and Analysis of Case}

The above two examples are modeled and predicted using BP neural network. Firstly, the model is repeatedly trained and corrected through the training samples, and then the future data is predicted by rolling in the future time points. The prediction results of two examples are shown in figures 3 and 4 respectively.

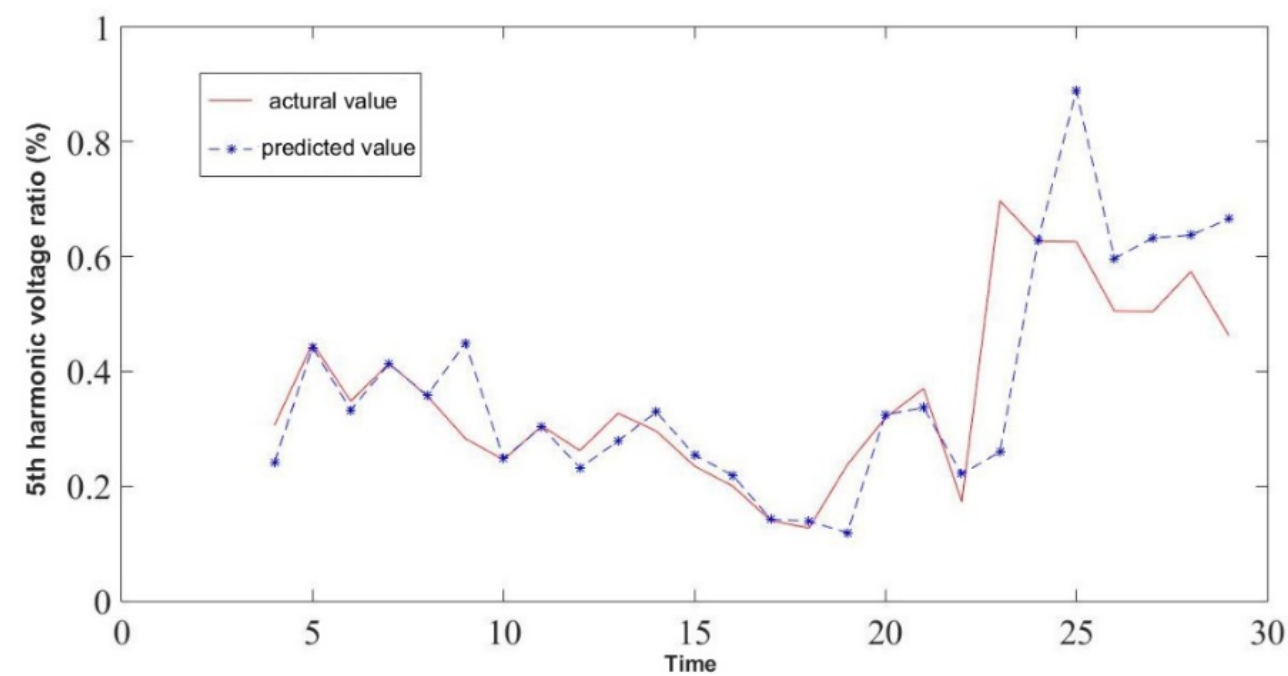

Figure 3. Comparison between actual value and predicted value of the 5th harmonic ratio. 


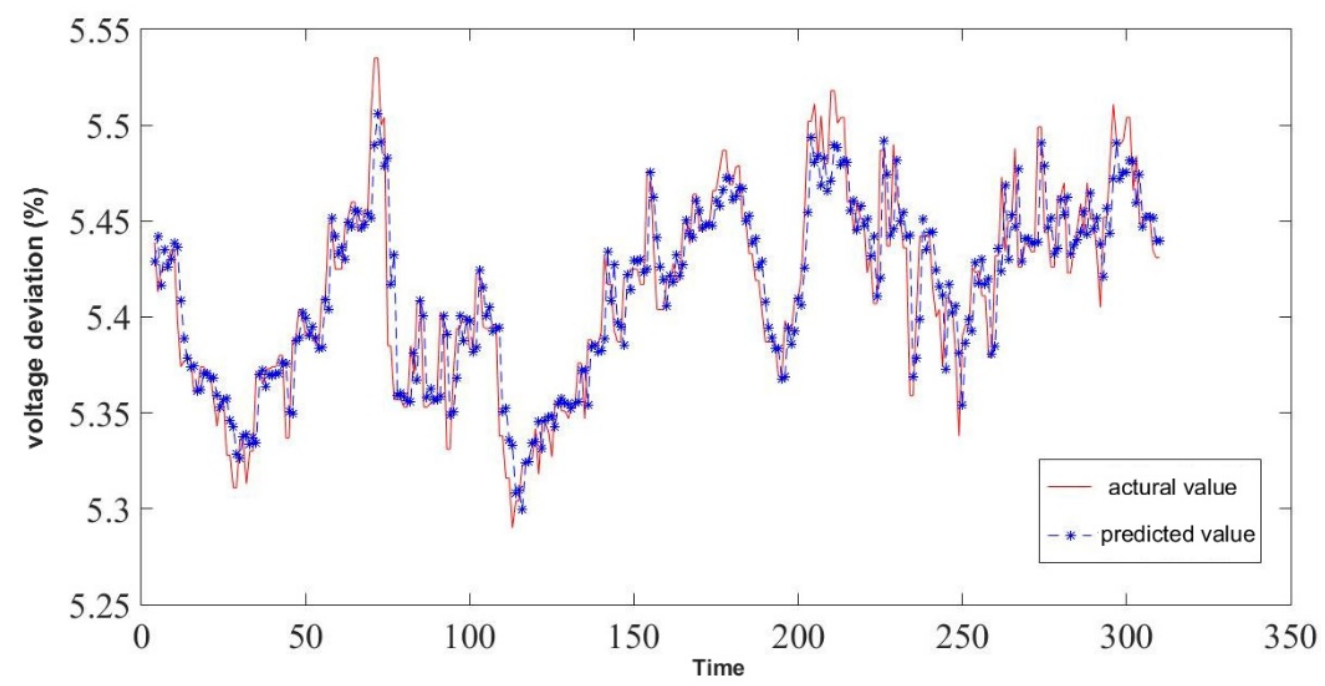

Figure 4. Comparison of the actual value and the predicted value of voltage deviation.

The errors between the predicted value and actual value of the two examples are calculated, as shown in table 1 and table 2 respectively.

Table 1. Predicted value and error value of 5th harmonic voltage ratio.

\begin{tabular}{cccc}
\hline Time points & Actual value (\%) & Predicted value (\%) & Error value (\%) \\
\hline 26 & 0.505 & 0.597 & 18.2 \\
27 & 0.504 & 0.633 & 25.6 \\
28 & 0.574 & 0.638 & 11.1 \\
29 & 0.462 & 0.666 & 44.1 \\
\hline
\end{tabular}

Table 2. Predicted value and error value of voltage deviation.

\begin{tabular}{cccc}
\hline Time points & Actual value (\%) & Predicted value (\%) & Error value (\%) \\
\hline 1 & 5.504 & 5.482 & 0.40 \\
2 & 5.466 & 5.481 & 0.27 \\
3 & 5.484 & 5.459 & 0.46 \\
4 & 5.449 & 5.474 & 0.46 \\
5 & 5.449 & 5.447 & 0.04 \\
6 & 5.454 & 5.452 & 0.04 \\
7 & 5.454 & 5.452 & 0.04 \\
8 & 5.435 & 5.451 & 0.29 \\
9 & 5.431 & 5.440 & 0.17 \\
10 & 5.431 & 5.440 & 0.17 \\
\hline
\end{tabular}

According to the figure 3 and table 1, it can be concluded that in the first example, the established neural network model has a better fitting effect on the training samples, but the prediction error on the predicted samples is larger. It can be seen from figure 4 and table 2 that in the second example, the established neural network model has better fitting effects on the training samples and prediction effects on the predicted samples. The neural network model accurately fitted and predicted the trend of data variation, and the relative error value between predicted and actual value were all below $1 \%$.

A comprehensive analysis of two examples shows that the training of neural networks requires a large data set. The prediction effect on the smaller data samples is poor; but on the larger data 
samples, after multiple model training and rolling prediction, the prediction effect is greatly improved, and the prediction results are more accurate.

\section{Conclusion}

This paper introduces the purpose and significance of the research firstly, then theoretically analyzes the BP neural network and the power quality prediction model based on this algorithm; finally, the method is verified by two examples. The power quality index numerical prediction model based on BP neural network needs to use a large amount of historical data for training, and then use the trained model to predict. The case study shows that the power quality prediction model based on BP neural network algorithm has good prediction results and high prediction accuracy when there are lots of training samples.

\section{References}

[1] Q. Li, Research on the method of power quality change trend forecasting and early-warning and its application, D. Guangzhou (2015)

[2] Z.J. Ding, P. Ling, S. Ouyang, J. Zeng, Mechanism of power quality forecast and early warning and their application, J. Ei. Commun. 27 (10) 87-92.

[3] H.T. Qing, J.Z. Li, Y. Liu, X. Dong, Prediction of the quality caused by electrified railways based on Monte Carlo, J. Ei. Commun. 39 (13) 64-70.

[4] W.W. Su, S.X. Ma, L.H. Qi, Predicting of power quality steady indicators based on ARIMA and neural network, J. Ei. Commun. 24 (3) 163-167.

[5] L. Huang, H. Chen, Q.M. Yi, Y.L. Tao, Load forecast based on hybrid model with k-means clustering and BP neural network, J. Ei. Commun. 37 (1) 56-60.

[6] L. Zhang, Study of the time series forecasting algorithm and system realization based on the ARIMA model, D. ZhenJiang (2008) 